\title{
Le paradoxe du torball : contribution à l'étude de l'intégration des déficients visuels
}

Odile Deleuze et Gilles Bui-Xuân

\section{(2) OpenEdition}

1 Journals

\section{Édition électronique}

URL : http://journals.openedition.org/corpsetculture/784

DOI : 10.4000/corpsetculture.784

ISSN : $1777-5337$

Éditeur

Association Corps et Culture

\section{Édition imprimée}

Date de publication : 1 juin 1998

ISSN : 1268-5631

\section{Référence électronique}

Odile Deleuze et Gilles Bui-Xuân, « Le paradoxe du torball : contribution à l'étude de l'intégration des déficients visuels », Corps et culture [En ligne], Numéro 3| 1998, mis en ligne le 04 mai 2007, consulté le 08 septembre 2020. URL : http://journals.openedition.org/corpsetculture/784 ; DOI : https://doi.org/ 10.4000/corpsetculture.784

Ce document a été généré automatiquement le 8 septembre 2020.

(c) tous droits réservés 


\title{
Le paradoxe du torball : contribution à l'étude de l'intégration des déficients visuels
}

\author{
Odile Deleuze et Gilles Bui-Xuân
}

1 Cette recherche a pour origine l'expérience d'une enseignante d'EPS en institut pour aveugles et amblyopes à Marseille. Partant du constat que ces jeunes gens sont souvent en échec scolaire et social, exclus, isolés, en souffrance, elle s'interroge sur la pertinence des contenus didactiques traditionnels au regard de leur relatif isolement, et leurs difficultés d'accès à une culture commune. Comment une société peut-elle intégrer ses déficients visuels? Quels liens peut-elle établir, qui soient suffisamment mobilisateurs pour un groupe social qui court sans cesse le risque de la marginalisation?

2 Une proposition de réponse est apparue avec l'ouverture d'un club de torball. Mais celle-ci ne va pas de soi, puisqu'elle mise sur une activité « ségrégative " pour créer du lien social en faisant le pari, à terme, de l'intégration. Trois ans après son démarrage, le club est omnisports et il est ouvert aux élèves de l'établissement, aux personnes extérieures et aux membres du personnel. Sur 61 licenciés, les trois quarts sont des déficients visuels qui jouent au torball, 8 font aussi du judo, et 43 d'entre eux sont inscrits en compétition d'athlétisme. $32 \%$ des membres du personnel y pratiquent de la gymnastique, du tir à l'arc ou du volley. En ce qui concerne le torball, en 3 ans, le nombre de torballeurs est passé de 14 à 46 , et $76 \%$ sont inscrits en compétition (scolaires ou divisions nationales). Deux filles sont en équipe de France, dont une a le statut de «sportif de haut-niveau ». 55\% des joueurs sont des élèves de l'établissement et $13 \%$ sont des anciens élèves.

3 L'objectif est de montrer le paradoxe de la pratique du torball, où les joueurs passent tout d'abord par une étape ségrégative et fortement identitaire puisque les sports collectifs des voyants ne leur sont pas accessibles, pour entrer ensuite dans un processus de légitimation et donc d'intégration grâce à l'appropriation d'attributs 
sportifs légitimes et aux changements sociaux et relationnels que cette démarche entraîne.

Le développement relationnel de l'enfant déficient visuel ou non-voyant

4 Les travaux de Y. Hatwell (1966) ont mis en évidence que la privation sensorielle, lorsqu'elle survient tôt dans l'enfance, altère le processus d'accommodation dans les relations objet/sujet. Bien que ce dernier détienne tous les éléments intellectuels nécessaires pour s'approprier le monde, il lui manque (es moyens de contrôle objectif pour s'ajuster au réel. L'enfant manifesterait alors des difficultés à s'adapter et à intérioriser des éléments de son environnement.

Le bébé aveugle montre dès l'âge de trois mois un important déficit tonique. L'absence des regards encourageants de la mère, le peu de sollicitation de son environnement, et surtout l'impossibilité d'imiter, entrainent un retard dans le développement moteur et des troubles dans l'acquisition du schéma corporel.

C. Schepens (1992) évoque au 2e Congrès Européen GRICCA le problème du verbalisme chez les non-voyants, mais aussi chez les déficients visuels, qui serait la conséquence d'un manque de relations entre le signifiant et le signifié.

7 La cécité provoque aussi des troubles psychiques par l'altération du contact de l'enfant avec le monde extérieur (introversion), la réduction des stimulations perceptives de son environnement sur sa personne, la diminution de son autonomie, de ses déplacements, ainsi que des limitations professionnelles et sociales et de fortes perturbations affectives. De plus, certains facteurs sociaux défavorisent les aveugles, tels que la vie en internat en établissement spécialisé, le rejet de la part de la famille, ou sa surprotection, la fréquentation scolaire chaotique en raison de nombreuses interventions chirurgicales, etc.

8 L'enfant aveugle ou déficient visuel sévère est confronté à une absence totale ou partielle des signes visuels de communication, d'accès à la culture sociale, du langage corporel. Si le geste et la parole sont très évocateurs socialement, le déficient visuel ne maitrise pas toujours la convention gestuelle ou la méconnaît. Il a ainsi souvent une vision erronée ou très éloignée de la réalité (Bullinger A., 1992).

Chez ces jeunes gens, l'incompréhension de l'entourage, les prévenances excessives, leur trop grande dépendance des autres malgré des efforts pour être autonomes et adaptés, rendent pesantes et pénibles l'absence ou l'altération grave de la vision.

Tous ces effets sur la vie sociale et relationnelle rendent l'intégration sociale difficile. Comment en effet un lien social pourrait-il s'établir avec des personnes qui n'auraient pas l'expérience ni la maîtrise de la relation sociale?

La reconnaissance sociale des minorités

11 Moscovici insiste sur l'importance de la mobilisation de toute minorité pour sa reconnaissance sociale par la majorité. "Pour que la minorité consistante acquière cette image positive, il lui suffit simplement d'être active et de faire connaître son existence » (Moscovici S., 1991 : 219). Il souligne le paradoxe des points de vues de toute majorité vis-à-vis des minorités. Bien que reconnaissant les qualités et les mérites d'un sous-groupe, une majorité a tendance à le mettre à l'écart de la vie sociale. Mais cela importe peu à cette minorité d'être rejetée car sa priorité est surtout "d'exister » et d'obtenir la reconnaissance de son existence. Elle va mettre tout en oeuvre pour assurer sa visibilité et son attirance et prouver « son aptitude à agir et à faire quelque chose vis-à-vis des autres » (Moscovici S., ibid.), 

composent. Afin de mieux les appréhender Didier Anzieu centre son approche psychanalytique du fonctionnement de groupe sur la notion d'imaginaire groupal et part de l'analogie entre le groupe et le rêve. Cela l'amène à décrire les processus imaginaires qui structurent la vie des groupes et qui fondent cet imaginaire groupal: l'illusion groupale, « le groupe-bouche », les fantasmes de casse, « le groupe-machine », la résistance paradoxale autodestructrice, les perturbations dues aux réminiscences de l'image paternelle ou du Surmoi. Anzieu compare le groupe à une enveloppe permettant de faire tenir ensemble les individus. Cette enveloppe " groupale " (trame symbolique) se compose d'un système de règles organisées en un réseau qui regroupe les pensées, le langage, les actions. Il donne la possibilité au groupe de se créer un espace interne lui procurant un sentiment de liberté, maintenant une communication et une temporalité propre (son histoire, son avenir, ses buts).

Sport et affiliation

R.-D. Alderman (1983) s'intéresse au besoin d'affiliation sociale. Ce besoin ne peut être satisfait que par une forme de contacts particuliers tels que l'appartenance, des relations fortes, le prestige à ses propres yeux grâce à l'appartenance au groupe de pairs, le statut, l'auto-appréciation, mais aussi l'angoisse de l'isolement. La pratique sportive permet-elle au sujet de soulager son angoisse en faisant partie d'une équipe ? Les besoins d'auto-appréciation, de comparaison et d'affiliation seraient satisfaits à condition que les sujets, et leurs réactions émotives, soient assez semblables. Le trait dominant dans les groupes reste toutefois l'importance de l'évaluation de son statut vis-à-vis de ses pairs.

Selon l'approche psychanalytique de M. Levêque et J.-R Heuzé (1995), le groupe de pairs est vécu par le sportif comme une référence et une protection maternelle, engendrant des processus identificatoires. Rassemblant alors toutes les déceptions et les frustrations de chacun, ce groupe devient un repère rassurant, réconfortant où peuvent s'exprimer les ressemblances identitaires. Puis, ces ressemblances une fois intériorisées par les individus qui le composent, conduisent à une conscience collective. L'organisation de cette vie psychique s'expliquerait par l'hypothèse narcissique. Grâce à l'énergie libidinale du sportif, le narcissisme permettrait une représentation idéalisée de soi, le désir de toute-puissance, le dépassement (Labridy F., 1993). De plus, l'entraîneur serait la projection de son Moi idéal narcissique et la structure sportive entretiendrait une représentation héroïque de soi, l'illusion d'une toute-puissance, la conviction d'être réellement soi-même.

15 Malgré les conséquences spécifiques de la déficience visuelle, et notamment la privation de toute possibilité de pratiquer des activités sociales valorisantes, comme les sports collectifs, et malgré la forte propension des aveugles à se sentir déconsidérés, leur tendance à se regrouper entre pairs n'ira pas dans le sens d'une marginalisation du groupe, mais au contraire dans celui de l'organisation d'une riposte collective sur le terrain même de l'excellence corporelle.

Construction dynamique de l'identité d'un groupe minoritaire par les sports collectifs

16 J.-P.Garel et M.Siros (1992) ont mis en évidence que l'enseignement des sports collectifs chez les déficients visuels et non-voyants permettait le développement des ressources perceptives et cognitives, la construction d'un espace d'action et d'un espace représenté ainsi qu'une concentration soutenue. De plus, les interactions et les échanges sont multiples car les joueurs doivent se mouvoir sur un même espace en

Corps et culture, Numéro 3 | 1998 
coopération constante, tout en élaborant des stratégies communes et donc en rompant avec leur propre isolement pour une organisation de l'équipe. Ils éprouvent des émotions collectives avec un plaisir partagé en cas de victoire, mais acceptent aussi la défaite de façon solidaire. Leurs travaux montrent que la pratique de sports collectifs développe des caractéristiques et des compétences spécifiques auprès des sujets nonvoyants et malvoyants :

- capacités d'exploration actives et d'action, de représentation du monde dans sa globalité ;

- modalités sensorielles, appelées suppléances sensorielles : le toucher, la proprioception, l'audition, la représentation spatiale, l'écholocation notamment;

- le joueur élabore des stratégies personnelles et collectives grâce à ses représentations, sa mémoire, ses capacités psychomotrices, sa communication avec les partenaires, son évaluation du jeu adverse et son niveau de compétence.

Cohésion et identité

"Une équipe sportive est un collectif d'individus qui possèdent une identité collective, qui ont des buts et des objectifs communs, qui partagent un sort commun, qui développent des modèles structurés d'interaction et des modalités de communication, qui manifestent une interdépendance personnelle, et à la tâche, une attraction interpersonnelle réciproque et se considèrent eux-mêmes comme un groupe » (Carron, cité par A. Marcellini 1995).

Les travaux de S. Moscovici (1991) révèlent que la cohésion est plus forte lorsque des personnes se croient semblables. L'importance de cette union crée la force du «nous » du groupe. La non-différenciation entre les individus entraîne des rapports de dépendance. Ce besoin des autres, et plus particulièrement chez les sujets ayant des perturbations ou des souffrances psychiques, explique en partie les effets d'influence sociale au sein du groupe. Il note que le degré d'importance de la cohésion donne la force du dynamisme de la minorité active.

Les études sur des sujets pratiquant un sport collectif différencient dans ce concept de cohésion, la cohésion sociale et la cohésion fonctionnelle. D'autres travaux distinguent l'intégration au groupe de l'attraction pour le groupe (Marcellini A., 1995).

Mais on ne peut parler d'identité et de handicap sans se référer aux travaux de E. Goffman (1963). Il distingue à propos du concept d'identité, l'identité sociale, l'identité personnelle et l'identité pour soi ou «identité sentie». En ce qui concerne l'identité sociale il dissocie l'identité sociale virtuelle (attributs et catégories imputés à l'individu par supposition) de l'identité sociale réelle (attributs et catégories d'appartenance de l'individu). Goffman oppose à ces deux concepts celui d'identité pour soi qui serait la représentation subjective de soi mais que la personne perçoit comme telle. Dès 1963, il s'intéresse aux individus ayant un handicap et aux conséquences sociales qu'il engendre. Il désigne sous le terme de «stigmate » la griffe, la marque négative en fonction du contexte que provoque le handicap : « Le mot stigmate servira (donc) à désigner un attribut qui jette un discrédit profond, mais il faut bien voir qu'en réalité c'est en terme de relations et non d'attributs qu'il convient de parler » (Goffman E., $1975: 13$ ).

«La première atteinte d'un individu présentant un handicap est sociale et inhibe ses élans de communication, ses désirs de socialisation, ses possibilités d'intégration. Il ne pourra être intégré qu'en cherchant à gommer son handicap bien que toute sa vie il gardera la marque qu'on lui aura imposée » (Stiker H-J., 1982). 

s'effectue essentiellement entre identité sociale et identité personnelle indifféremment appelée aussi individuelle ou identité pour soi: "Mon identité c'est donc ce qui me rend semblable à moi-même et différent des autres, ce par quoi je me sens exister en tant que personne et en tant que personnage social (rôles, fonctions), ce par quoi je me définis et me connais, me sens accepté et reconnu comme tel par autrui, mes groupes et une culture d'appartenance » (Tap P., ibid. : 8).

21 Ainsi l'appartenance à un collectif poursuivant un but commun, à une équipe sportive, mobilise un système primaire de cohésion sociale, et procure aux torballeurs un sentiment global d'identité sociale. La démarche collective de déstigmatisation (Marcellini A., 1991) va les confronter alors aux difficultés réelles de l'étayage du lien social.

Pratique du torball et processus de légitimationPrésentation du torballHistorique et règlements développé en Allemagne à partir de 1955 dans les centres de rééducation d'invalides de guerre. Dans les années 1970, cette activité n'est pratiquée régulièrement que dans quelques clubs français (Thionville, Metz, Strasbourg) qui rencontrent très fréquemment des équipes allemandes, suisses puis italiennes. Les premiers championnats de France apparaissent dans les années 80. La pratique du torball s'étend de plus en plus en Europe. Mais il faut attendre 1988 pour que l'IBSA (International Blinded Sport Association), dont dépend la Fédération Française Handisport (sports pour les personnes ayant un handicap physique ou sensoriel), s'y intéresse. En effet, bien que le torball soit une discipline très largement pratiquée en Europe, le goal-ball (d'origine américaine) est le sport collectif reconnu et imposé dans les «jeux paralympiques » et le plus structuré sur le plan international. Mais il est rarement pratiqué dans les clubs français car peu apprécié par les joueurs qui lui préfèrent le jeu dynamique et spectaculaire du torball.

Depuis 1988 il existe: un règlement international de torball; des compétitions officielles internationales, européennes; des sportifs de haut niveau sélectionnés, soit en équipe de France masculine, soit en équipe de France féminine; des joueurs reconnus «élites » et inscrits dans les registres du Ministère de la Jeunesse et des Sports.

Le jeu de torball fait partie des jeux collectifs qui se jouent entre deux équipes opposées avec un règlement international standardisé. Le terrain de torball fait 16 mètres de longueur sur 7 de largeur, et la ligne de fond représente la ligne de but (la victoire se joue au score, au terme du temps réglementaire: l'équipe gagnante est celle qui a marqué le plus de buts). La cage fait 1,30 mètres de hauteur. Une corde, à laquelle sont accrochées des clochettes, est tendue d'un côté à l'autre du terrain à une hauteur de 40 $\mathrm{cm}$, séparant le terrain en deux parties. Deux cordes, également avec des clochettes, sont fixées de part et d'autre de la corde centrale, laquelle délimite les surfaces des deux équipes -chacune de 6 mètres sur 7- où se placent les joueurs. Trois petits tapis sont placés sur le sol de chaque côté, pour que les joueurs -dont les yeux sont bandésaient un point de repère. Chaque équipe a trois joueurs. Le ballon utilisé contient un petit grelot à l'intérieur. Le son émis par ce grelot permet aux joueurs de situer le ballon de façon précise. Il a un diamètre de 65 à $69 \mathrm{~cm}$ et ressemble à un ballon de volley-ball. Chaque partie dure deux mi-temps de cinq minutes chacune, et est dirigée

Corps et culture, Numéro 3 | 1998 
par un arbitre, un chronométreur, un marqueur et quatre juges de touche. Le temps doit être arrêté, en cas de remplacement, de hors jeu, de coup-franc, de penalty et d'arrêt de jeu décidés par l'arbitre.

Les catégorisations de la Fédération Française Handisport

Les catégorisations de la FFH pour les déficients visuels sont celles de l'IBSA qui élaborent les règlements sportifs internationaux :

- B1 : depuis la cécité totale à la perception de la lumière, sans distinguer les contours et les formes ;

- B2 : acuité visuelle inférieure ou égale à $2 / 60$ e et un champ visuel inférieur à 50 ;

-B3 : acuité visuelle jusqu'à 6/60e et/ou un champ visuel maximal de 200 .

Mais B1, B2 et B3 peuvent pratiquer ensemble, tous ayant les yeux bandés.

Ainsi, les licenciés ayant une vision supérieure à 1/10e, ne peuvent prétendre concourir dans les championnats officiels Handisport, mais sont toutefois admis pour pratiquer les activités dans le cadre de leurs loisirs.

Analyse historique et institutionnelle du torballLe torball, une pratique spécifique et ségrégativeUne première période très fermée

En 1955, le torball n'est pratiqué que dans des Centres d'invalides (aveugles) de guerre en Allemagne. Dans le début des années 1970, alors que les clubs de torball regroupés dans l'Association des Aveugles d'Alsace et de Lorraine se rencontrent et participent régulièrement à des tournois en Allemagne, aucune tentative n'est faite pour développer cette pratique dans les autres régions de France. Ces groupes sportifs rassemblent des adultes issus souvent d'associations très fermées pour personnes aveugles (la Croisade des Aveugles, l'Association Valentin Haüy...).

Deuxième période : une pratique moins cloisonnée

En 1983, le Championnat de France se déroule à Thionville. La plus jeune équipe du tournoi (entre 15 et 20 ans) est celle d'Arc-en-Ciel. C'est le seul club parmi les dix, à réunir pour la première fois des élèves non-voyants d'un établissement spécialisé. Deux équipes comportaient des « féminines ».

Après ces championnats, des tournois promotionnels inter-régionaux sont organisés.

Une étape de reconnaissance sociale

Une Commission Nationale torball/goalball se structure au sein de la Fédération Handisport. Elle crée et met par écrit un règlement officiel pour les Championnats de France masculins et féminins puis définit ses diverses missions.

En 1986, constamment sollicitée par les fédérations des pays européens (Allemagne, Autriche, Belgique, France, Italie, Pologne, Roumanie, Suisse), l'I.B.S.A statue enfin sur un règlement international uniforme de torball et limite l'accès aux compétitions aux déficients visuels ayant un degré visuel maximum de $1 / 10^{\circ}$ et un champ de $20^{\circ}$.

Des équipes nationales de torball peuvent se constituer enfin et disputer les Championnats d'Europe, les Championnats du monde, et les clubs champions nationaux ont la possibilité de concourir aux Coupes d'Europe des clubs champions. Le goalball n'a pas ces officialisations. Toutefois, il devient le sport collectif paralympique des déficients visuels après quelques modifications de son règlement pour les Jeux Paralympiques aux Etats-Unis en 1988. En effet, le torball était trop « européen ». Mais en France, les Jeux du Pacifique et les Jeux de l'Avenir s'ouvrent au torball et la 
Commission Nationale Handisport des Sports pour déficients visuels s'engage à aider les clubs et les sportifs (dans la mesure de ses moyens).

L'étape de légitimation

Au début des années 1990, les associations et les équipes se multiplient et une dizaine d'établissements scolaires spécialisés participent au premier Critérium National Scolaire de torball pour les catégories de moins de 20 ans, soit une cinquantaine de participants (en mars 1997: 110 participants). Parallèlement, lors des Championnats de France ou des tournois pour des journées sportives importantes, les média (télévision, journaux, magazines) commencent à S'intéresser à ce sport qui leur paraît "insolite ", "spécial », mais « sport comme les autres sports »...

Des stages nationaux de présélection s'organisent régulièrement trois fois par an.

Après les Jeux Paralympiques de Barcelone de 1992, un projet fut élaboré entre Handisport et le Ministère des Sports pour reconnaître et aider les sportifs de haut niveau handicapés. Les critères de sélection sont identiques à ceux des voyants.

Aujourd'hui, les clubs de torball "ayant fait leur preuve" (résultats, nombre de licences, actions phare ...) perçoivent, comme les clubs de valides, des subventions supplémentaires pour l'organisation de Championnats de France ou internationaux, pour les déplacements de championnats officiels ou l'achat de gros matériel (Fond National pour le Développement du Sport, Municipalité, Conseil Régional, Conseil Général).

Conclusion provisoire

38 L'analyse historique et institutionnelle du torball confirme que le paradoxe social et collectif du torball apparaît dans son évolution historique : d'une activité ségrégative elle se transforme en une activité spécifique mais légitime. Le torball est désormais inscrit sur la liste des disciplines sportives reconnues par le Ministère des Sports, au même titre que celles des voyants. Cet événement capital permet d'intégrer des sportifs déficients visuels dans des groupes de sportifs de haut niveau valides. Le regard des autres et la considération des instances officielles en ont été assurément modifiés.

Les torballeurs

39 Les licenciés, ayant choisi cette activité dans le cadre de la compétition, y côtoient des partenaires, des adversaires, des entraîneurs, des arbitres, le public et parfois les média. Ils peuvent jouer en fonction de leur niveau de jeu et non pas en fonction de leur degré de handicap. L'aspect sportif du torball apporterait-il un intérêt d'identité de groupe de personnes déficientes visuelles ou aveugles pratiquant un sport, venant en opposition aux stigmates de ceux qui ne sont que mal-voyants ou non-voyants ? Ce phénomène de groupe serait-il moins discrédité que l'autre ? Pour les individus qui le composent il participerait d'un processus de diminution des stigmates. L'attribut de "groupe de sportifs» ajouté à celui «d'aveugles» améliorerait-il leur sentiment d'identité sociale?

Quel est l'intérêt social mis en jeu dans la composition de ce sous-groupe de torballeurs les rassemblant dans un même destin collectif?

41 Pour David Le Breton (1995), les Activités Physiques et Sportives (APS) ne peuvent avoir d'impact sur les stratégies d'insertion sociale et de restauration identitaire des personnes dites handicapées que si elles ont du sens pour ces individus.

De nos jours, les activités physiques s'adressent à tout le monde, y compris aux personnes qui ne correspondent pas aux normes physiques, mentales et sociales. Les 
APS n'ont pas de vertu en soi d'intégration ni de socialisation ni de renforcement du lien social. Toutefois, elles permettent une construction ou une reconstruction identitaire des personnes reconnues handicapées par la société. La personne stigmatisée va s'approprier ou se réapproprier son image corporelle, affirmer son estime de soi, construire ou réactualiser puis dynamiser son projet de vie, harmoniser ses représentations avec celles du monde. Les APS utilisées alors comme moyens par les " éducateurs » sont un tremplin, voire une aire transitionnelle au sens de Winnicott où les personnes stigmatisées rencontrent les « valides ». Elles sont toutefois adaptées.

«[Le sport] est malgré ce que l'on peut en dire par ailleurs, un formidable creuset identitaire qui dans notre civilisation passe toujours par le corps. » (Broyer G., 1995 : 170)

Mais le torball ne peut être considéré comme une activité physique adaptée au sens propre du terme, car il est important de relever le caractère spécifique de cette discipline, conçue pour et par des déficients visuels fortement handicapés et portant tous un masque opaque. Son essence est unique et originale bien qu'il existe des analogies avec des APS collectives des personnes voyantes. Les joueurs sont cependant imprégnés par la culture "légitime » même s'ils en sont exclus. Ils cherchent alors à adhérer à quelque chose qui appartient à cette culture en tant qu'objet culturel, mais qui leur est propre et qui est créé spécialement pour eux. Ils tentent de démontrer que le torball est un réel objet culturel tout en se ré-identifiant.

On peut considérer tout d'abord que ce besoin d'identité d'un groupe qui cherche à pratiquer une activité fermée aux autres rejoint un processus de ségrégation. Mais, par la suite, le fait d'avoir accès à la performance, de militer pour la reconnaissance de cette activité collective et spécifique en tant qu'entreprise culturelle minoritaire et identitaire est une démarche intégrative, une étape préliminaire et nécessaire pour accéder à la culture "légitime». Ces jeunes déficients visuels n'ont plus alors d'appréhension de se faire appeler "torballeurs" car le torball est un tremplin de reconnaissance sociale. En effet, ils peuvent entrer dans l'espace social et s'approprier des fondamentaux déterminant la culture sportive légitime puisqu'il revêt tous les attributs d'un sport collectif de voyants (terrain délimité, ballon, but à atteindre, partenaires, adversaires, arbitre, règlement institutionnalisé et international, titres officiels, sportifs de haut niveau). En confirmant sa reconnaissance, la société va leur donner les mêmes attributs positifs qu'aux sportifs voyants. Ils accèdent alors à une activité culturelle valorisante et ainsi intégrative.

Puisque la valeur idéologique "travail» est problématique dans notre société, les personnes atteintes d'un handicap revendiquent d'autres valeurs : le sport, la qualité de vie, les loisirs... Aussi, la pratique spécifique du torball dans l'association sportive regroupant des déficients visuels sportifs serait, par la mise en place d'un processus d'identification groupale conscient ou inconscient, une solution stratégique, directe ou indirecte et à court ou long terme, aux difficultés existentielles diverses (individuelles et communautaires), et ceci, grâce aux interactions personne/groupe. Leur nouveau statut changerait le regard des autres, et leur identité personnelle se renforcerait grâce à la construction d'une identité collective.

Hypothèse générale: paradoxe individuel, paradoxe social

Cette recherche se propose donc de mettre en évidence un processus paradoxal d'intégration réussie. L'hypothèse est que l'intégration pourrait passer par la 
construction préalable d'une activité spécifique, voire ségrégative, qui une fois reconnue et légitimée, offrirait à ses adeptes un tremplin vers l'intégration.

Ce processus paradoxal se développerait à deux niveaux : sur le plan social, collectif et institutionnel, l'histoire du torball permet d'illustrer comment une activité "ségrégative» peut se transformer en activité légitime; mais cela ne serait pas possible si, sur le plan individuel, une mutation ne s'opérait pas dans la quête identitaire des pratiquants qui, d'abord attirés par la spécificité du torball, ne revendiqueraient bientôt la reconnaissance d'une identité de sportifs légitimes, à l'image des valides.

Une étude de cas singuliersPrésentation de la population étudiée

Sur les 46 torballeurs licenciés au club FFH., plus de la moitié ont au moins 18 ans. Les 12 sujets adultes choisis répondent aux critères communs suivants : sexe : masculin ; âge. entre 19, 5 ans et 27 ans ; ancienneté au torball : entre 2 ans et 10 ans; degré de vision : de la cécité totale à $1 / 10^{\circ}$ de vision, soit les catégories de la FFH et de l'IBSA : B1, B2, B3, niveau d'étude égal ou supérieur à la classe de cinquième : orientation vers le diplôme de CAP ou classe de troisième; tous élèves ou anciens élèves de l'institut Régional des Jeunes Aveugles 1 le nombre d'entraînements : une fois par semaine pour 2 sujets inscrits en Loisirs et deux fois pour les autres inscrits en compétition.

Organisation du travail méthodologiqueLa méthode des cas singuliers

La méthode de cas singuliers a été retenue car elle permet d'appréhender le monde subjectif des déficients visuels ou non-voyants, très différents dans leur handicap, leur pathologie, leur milieu social, leur culture, leur religion, leur histoire et leur souffrance personnelle, mais présentant toutefois des régularités dans leur façon d'appréhender leur handicap, leur vie dans la société et leur façon d'améliorer leur existence.

Deux types d'outils ont été utilisés

- l'analyse historique et institutionnelle du torball où l'évolution de l'étape ségrégative vers

l'étape intégrative a déjà été développée ;

- la technique de l'entretien non-directif où les contenus seront analysés à partir des indicateurs et les thèmes les plus fréquents et les plus importants pour les sujets.

La sélection des indicateurs et des indices

51 Après lecture des douze entretiens, trois indicateurs et leurs indices permettent de révéler les différentes étapes qu'ont traversé les sujets :

- $1^{\mathrm{e}}$ indicateur la quête identitaire comme processus ségrégatif.

- $2^{\mathrm{e}}$ indicateur la quête de reconnaissance sociale du groupe ; le groupe comme source

d'influence sociale.

- $3^{\mathrm{e}}$ indicateur $:$ la quête de légitimation et le processus d'intégration.

Analyse des entretiens

Etape 1 : le processus ségrégatif.

Indicateur 1 - la quête identitaireL'identité personnelle« l'exclusion de la culture sportive légitime »

Les déficients visuels et les non-voyants se situent dans le même système social, dans la même culture sportive que les sportifs voyants. Mais la déficience visuelle, entraînant une incapacité fonctionnelle, les empêche de réaliser des performances équivalentes à celles des "valides", et remet en cause, voire interdit, leur intégration dans les clubs de sports collectifs ordinaires, et notamment de football. 

regrettent de devoir se regrouper entre eux ; ils désirent adhérer à une culture sportive qui leur soit accessible. Ils choisissent la solution de se licencier dans une association créée pour les déficients visuels. La distinction se fait alors par rapport à la notion de normalité car le milieu non-handicapé est appelé «normal » et pas " voyant ».

"J'ai essayé de m'intégrer et je me suis pris une claque.»

«Ce sport-là remplace un sport que je ne pourrai jamais faire : le foot. »

«Le club de torball est une structure posée qui permet à mon handicap de pouvoir

faire une pratique sportive, car il ne peut pas en faire dans un club normal. » « Le

torball est une activité sportive qui correspond à mon handicap.» «Je ne pensais

pas qu'il existait des structures adaptées comme celle-là. »

« la spécificité du torball»

Cette activité est spécifique et compense les sports qu'ils ne peuvent faire. Elle les rassure, les autorise à s'intégrer dans une équipe, dans un club, à y avoir leur place, à éprouver de fortes émotions, du plaisir, à acquérir la santé physique et morale. Adaptée à leurs possibilités et tenant compte de leurs limites, car conçue pour eux, elle les transforme et de plus leur appartient.

"Le torball est le seul sport collectif que je puisse faire et qui me convienne aussi. »

«Le torball c'est un sport pour moi, spécial pour ma déficience. » « Il est fait pour nous et on y vit tous ensemble. »

«la valorisation de soi »

La pratique du torball, en tant que processus dynamique, constitue un creuset identitaire pour ces jeunes gens et participe à un processus de transformation de leur «identité sentie», «identité pour soi». Le groupe a une forte influence sur leur personnalité. Il permet de relativiser leur handicap, le dédramatise. Ils reconstruisent alors une « image de soi » positive dans des situations valorisantes et gratifiantes. Ils se perçoivent autrement à travers ce sport collectif et le préfèrent aux sports individuels de valides qui peuvent pourtant être adaptés, et donc accessibles.

Cette pratique collective spécifique leur permet de s'enrichir sur le plan personnel. De s'estimer, de se savoir " capables de », les rassure et les aide à s'affirmer, à s'épanouir, à s'ouvrir progressivement vers l'extérieur et de changer.

«Le fait d'être ensemble au torball me renvoie une image de moi positive.» «Il m'aide à m'aimer » "Cela me revoie une image de moi enfin positive. »

" J'ai besoin de m'affirmer » "C'est pour moi une façon de m'épanouir » « Le torball permet de bouger et de s'extérioriser » «Le torball m'a fait quelque chose grâce à son ambiance différente. »

L'identité sociale« l'affiliation »

Il apparaît chez les 12 sujets interviewés un très fort besoin d'affiliation à un groupe de pairs. Les thèmes les plus fortement représentés sont le besoin d'appartenance, les relations fortes, le besoin de statut. 
professionnels, des personnalités ...) lors des tournois importants. Ils recherchent alors la visibilité, la reconnaissance statutaire de leur identité personnelle. Au sein de ces organisations collectives, ils rompent avec leur isolement social lors des compétitions. Cela leur donne la possibilité d'élargir leurs connaissances, d'entretenir d'autres échanges, d'élargir les limites de leur connaissance du monde. Ils reconnaissent leur handicap et ne veulent plus être ignorés des voyants. Grâce à leur pratique spécifique, ils veulent être connus et reconnus en tant que personnes portant un stigmate. Ils renforcent à la fois leur identité pour soi et leur identité sociale et se préparent insensiblement à franchir d'autres étapes.

«C'est important d'avoir un groupe pour faire le même sport, même en loisir et qui sont là pour se faire plaisir pour faire équipe. » "Il se passe quelque chose de fort entre nous. » «J'aime bien être connu et reconnu. »

«Les torballeurs je les considère comme moi-même." « On vit ensemble certains moments d'émotion, de désir de partage de choses communes.»

«Etre sur le terrain et mener une vie de club. » «Ce groupe s'est formé car on avait à peu près le même âge et on commençait a peu près ensemble. On formait la bande. " "On est une bande de copains sur et hors du terrain. " "Cette entente, cette ambiance s'est développée parallèlement à notre vie d'internat ou scolaire. » " On peut connaître des gens importants. » «Parler à des gens qui jouent au torball à Clermont, à Paris, parler avec des voyants, c'est une façon de me démarquer de dire que j'existe quoi !»

"J'aime communiquer et j'aime voyager»

"Agrandir mon réseau de connaissances. » «la communication»

61 Ils ont un fort besoin de communiquer dans l'affiliation au groupe de pairs pour mieux se connaître et s'identifier, pour créer des relations affectives intenses, pour se protéger, pour moins souffrir, pour se sentir plus valorisés. Ils évoquent ce besoin de communication comme une nécessité et une particularité spécifique aux torballeurs du club, un mécanisme de défense venant suppléer l'absence ou l'altération de la vue.

Le torball est leur «lieu secret », le club est leur monde commun qui leur appartient exclusivement, un espace de vie sacralisé. Ils reprennent le langage visuel où les mois sont imagés, colorés dans un environnement où ils parlent une langue faite par et pour les voyants. Espace de vie étranger mais dans lequel ils veulent s'intégrer pour vivre, mais où ils doivent fournir des efforts constants d'adaptation, voire de suradaptation pour les non-voyants de naissance.

«Le torball me permet de me faire connaître à l'extérieur en tant que déficient visuel»

"Je me sens bien sur le terrain quand j'arrive à donner des consignes, à bien communiquer avec les joueurs, a apporter quelque chose à l'équipe.»

"J'ai d'autres moyens d'échange, autres que le regard, ce sont des substitutions inconscientes. »

«Il existe certains mécanismes relationnels qui se déclenchent entre nous. »

«Avec les voyants autour d'une table je joue la carte de l'intégration, les mots ont plus de valeur que le regard, même si je sais qu'il peut être très significatif. »

« la socialisation»

Le club est une micro-société dans laque le la loi doit être respectée ainsi que les normes établies et imposées par le sous-groupe. Elle protège et rassure ceux qui les respectent et elle exclut ceux qui les bafouent et qui lui portent ombrage à l'extérieur.

"Ce qu'il y a dans le club, les règles et tout ça, cela me sert quelque part.»

"Ceux qui font les provocateurs, les "esbroufs", les gamineries tu dois les virer» 
L'identité collective du groupe « la fusion des individus »

Le groupe trouve son identité particulière en même temps que celle des individus qui revendiquent leur unité. Arrivé à ce dernier stade dans l'étape ségrégative, le groupe crée une enveloppe symbolique qui enserre tous les individus qui le composent. Ils recherchent la fusion. Deux faces sont alors nécessaires. L'une tournée vers l'extérieur sera appelée «l'union pour la force », l'autre vers l'intérieur, sera nommée «l'union pour la conformité et l'osmose.»

65 - « l'union pour la force.»

Les individus organisent au sein de ce groupe minoritaire et fusionnel leur défense collective dirigée vers la réalité extérieure par :

- une bande qui peut s'acharner contre ceux qui représentent un danger, qui veut vaincre grâce à une force unique ;

- un espace qui recueille toutes les énergies individuelles pour engendrer une force collective ;

- cette force collective, commune, influe à son tour sur chaque individu qui ressent une illusion de toute-puissance, et cet effet n'est possible que par le nombre restreint de ses membres (parité restreinte).

- «l'union pour la conformité et l'osmose.» Ils s'unissent pour être identiques, uniformisés, uniques.

Il se forme un état psychique du groupe, un «soi imaginaire groupai " par une forte identification des joueurs entre eux pour créer le joueur de torball unique.

«Les torballeurs c'est comme une meute.»

«Cette force au sein de l'équipe, c'est un peu comme un puzzle.»

«Tous ensemble on est vraiment plus forts.» «Dans le torball je ressens comme

une force."

«S'il existe cette notion de force entre nous, c'est qu'on est moins nombreux qu'en foot. "

«Il y a des joueurs identiques, l'entraîneur peut changer de pièces, on ne verra pas de différence. »

«Pour moi l'important c'est le groupe, la construction de ce groupe. »

"C'est important de pouvoir s'unifier entre nous.»

« la solidarité »

Le groupe a le devoir d'assurer en permanence une solidarité pour protéger et aider sans discontinuer tous ses licenciés.

Cette notion joue un rôle important dans l'affirmation de l'identité du groupe de torballeurs. Ses missions sont précises et se caractérisent dans :

- la protection de tous les joueurs

et

- la gestion de leurs problèmes.

"S'il y en a un qui "disjoncte", il faut qu'il y en ait au moins un qui soit là. C'est quelque chose qu'on doit faire à tour de rôle. »

«Il faut les encourager »

"On se soutient physiquement et moralement. »

"Si on baisse les bras, il y aura toujours quelqu'un qui aidera à les relever "

«la distinction avec les déficients visuels non torballeurs »

Ils se distinguent des autres déficients visuels et les considèrent différents d'eux, bien que certains entretiennent de bonnes relations. Ils perçoivent leur mal-être, leur difficulté à accepter leur handicap de déficients visuels, et pensent que le torball les 
aiderait sur le plan personnel et pourrait faciliter leur intégration au sein du groupe des déficients visuels.

«Avec les non-torballeurs, on se dit bonjour, bonsoir, on se connait comme cela.

Rien de spécial entre nous.» "Avoir de la complicité, c'est plus difficile avec quelqu'un qui ne connaît pas le torball. »

Etape 2: le groupe à la source d'un processus d'influence sociale.

Indicateur 2 - la quête de reconnaissance sociale du groupeLe militantisme

L'identité de groupe est enfin construite lorsque les individus ressentent tous leur union pour ne former qu'un seul et unique torballeur. Ils réunissent ainsi toutes les énergies, toutes les volontés, tous les fantasmes qui les animent. Fort de ses potentialités internes, de cette consolidation, le groupe s'ouvre vers l'extérieur avec consistance et assurance pour obtenir la reconnaissance de son existence à l'échelle nationale et internationale, et celle de ses capacités. Cette entreprise est difficile. Les torballeurs s'y engagent comme pour relever un défi, pour légitimer leur existence. $\mathrm{Ce}$ besoin de reconnaissance s'exprime à travers des conduites destinées à influencer les opinions des personnes voyantes. Alors, cette minorité active se mobilise et met en place des stratégies de militantisme en toute indépendance : visibilité, attirance, prises d'initiatives et échanges avec des personnes différentes.

«Dans le groupe des torballeurs, il y a le sport qui nous unit. C'est le sport le médiateur de tout ça. »

«Ce sport n'est pas encore tout à fait reconnu par les autres, et nous on essaie de le faire connaître et reconnaître partout en France et à l'étranger »

«On veut montrer aux voyants ce qu'on peut faire avec nous. Ce qu'on peut faire dans certains sports que très souvent ils ne connaissent pas. »

« la visibilité »

71 Les individus qui composent le groupe désirent être identifiés comme des déficients visuels particuliers : ils sont d'abord torballeurs.

Suite à leurs revendications de reconnaissance sociale convaincantes, les joueurs espèrent des perspectives intégratives à long terme. Ils se démarquent des déficients visuels qui n'agissent pas, qui subissent leur destin.

« Le torball peut nous aider à nous faire reconnaître pour notre intégration. » «l'attirance»

Cette minorité veut être individualisée. Elle montre ses mérites et ses compétences pour attirer non pas de la sympathie mais de l'estime, de l'admiration devant les performances réalisées, et tous les joueurs doivent contribuer à satisfaire cette tâche.

« Comment peuvent-ils espérer trouver un appart, des copains, s'intégrer quoi! »

«Le groupe de torballeurs sur le terrain c'est important à montrer car on montre qu'on est comme les valides. »

«les prises d'initiative»

Afin que le groupe renvoie une image positive et qu'il soit influent sur les représentations sociales d'autrui, les actions et les activités développées doivent prouver cette volonté, cette aptitude à agir. Il s'agit de parler, d'expliquer, de justifier, pour inciter la curiosité, le désir de connaître, mais aussi montrer et démontrer sur le terrain.

«Quand je fréquente les gens valides, j'essaie de parler du torball même si je le présente comme un sport pour handicapés visuels. Ils posent des questions et ils sont intéressés et ils viennent me voir des fois en tournoi. »

« la recherche d'échanges avec les gens différents » 
minorité veut être écoutée. Elle recherche les contacts et la communication auprès des personnes voyantes. La pratique du torball représente un support de conversation et un moyen d'influence sociale.

«Je peux communiquer grâce au torball avec toutes sortes de gens et réciproquement, ils peuvent aussi communiquer avec moi. »

Etape 3 : le processus de légitimation.

Indicateur 3 - la quête de légitimationL'adhésion à la culture sportive« l'assimilation des attributs de sports collectifs de voyants"

Les torballeurs reconnaissent le caractère singulier et spécifique du torball, peu connu par rapport à celui plus normalisant de certaines activités physiques adaptées (A.PA.).

Cependant, ils affirment l'appartenance du torball à la famille des sports collectifs du milieu ordinaire. Ils assimilent ses attributs à ceux des sports d'équipes de valides.

«Le torball équivaut à un sport collectif de valides.»

«Le torball est comme les autres sports collectifs.»

"Comme les autres sports, il y a une stratégie à élaborer, un côté intellectuel mais

aussi physique. C'est un peu les deux. »

«l'assimilation des attributs de sportifs voyants»

Leur unité, leur identité de groupe de torballeurs existe surtout dans leur volonté de légitimation et leur passion commune pour les sports collectifs. Leur cécité et leur déficience visuelle ne sont plus ce que l'on perçoit au premier abord.

«Actuellement, un lien existe entre nous tous, le sport avant tout. »

«l'assimilation des attributs de sportifs de haut niveau »

Les évaluations faites par les sélectionneurs nationaux sont d'autant plus importantes et valorisantes pour les torballeurs que ces évaluateurs sont voyants. Etre détectés et reconnus comme "élite " par des tests sévères et les critères non discriminants du milieu ordinaire, cela accentue leur sentiment d'appartenance au monde sportif de haut niveau des voyants.

L'analyse institutionnelle et historique du torball a permis d'énoncer les avantages et les honneurs auxquels ils peuvent prétendre lorsqu'ils sont cités par le Ministère des Sports comme «sportifs de haut niveau »: ils sont célèbres parmi les torballeurs français et étrangers, rencontrent des personnalités, reçoivent des distinctions et des bourses, sont invités régulièrement dans des protocoles officiels, sont intégrés dans le groupe des sportifs voyants de haut niveau.

81 Hors du contrôle du groupe, le joueur veille à conserver en toute circonstance son sérieux, son honorabilité et essentiellement le contrôle de ses affects. En effet, une incartade pourrait nuire a sa reconnaissance de grand sportif, inhiber l'exploitation de ses capacités, et dévoiler ses faiblesses psychiques, ce qui engendrerait de nouveau sa stigmatisation sociale et amoindrirait son intégration et la considération de ses attributs de sportifs de haut niveau.

« Pour être en équipe nationale, pour être sélectionné, pour être le meilleur »

"Viser le plus haut niveau même si je suis au top.»

«Si tu disjonctes, tu casses tout, tu détruis ton jeu mais surtout tu te détruis toi-

même »

«Maintenant que je me connais mieux, le torball va me permettre d'essayer un autre sport : de voyants.»

«Quand je regarde le foot à la télé, je me dis moi aussi j'ai mon sport, moi aussi je suis sportif.»

Le processus de changement« l'identité » 
De l'étape ségrégative, fortement identitaire, à celle de la légitimation, les joueurs sont conscients de leur progression, de leur changement :

- ils intègrent les règles de la vie sociale ordinaire et $y$ participent ;

- ils ont assimilé les attributs de la culture sportive des voyants ;

- ils se sentent de véritables sportifs.

Cette assimilation des propriétés du monde sportif des voyants déclenche chez les torballeurs de nouveaux processus identificatoires. Il n'existe plus chez eux ni exclusion, ni cloisonnement.

«Maintenant, je me connais plus, je connais mes possibilités, mes exploits sportifs. Le torball va me permettre de m'intégrer dans un sport et un club normal. » "C'est primordial le torball, c'est un sport trop bien pour moi, mais il faut maintenant essayer de faire quelque chose à côté. » «Pouvoir jouer le rôle de l'intégration du fait de le vouloir et d'être prêt à faire d'autres sports à côté. »

"Je veux que les autres s'intéressent à moi, il faut que j'aille au-delà de mon

handicap et avoir plus de facilité pour l'intégration. » "l'intégration»

4 Mieux se connaître et connaître ses capacités, sentir ses limites, évaluer ses progrès, adhérer à la culture sportive ordinaire sans l'ombre du stigmate, c'est réussir à se libérer de son handicap, c'est envisager enfin d'entreprendre des démarches pour s'intégrer.

La pratique du torball a permis progressivement le déclenchement de ce processus de transformation et d'élan vers les autres. Arrivé à l'ultime palier de leur quête de légitimation, cette activité représente pour eux un tremplin pour l'intégration. Ils sont enfin prêts pour la tenter et pour la réussir.

Commentaires

86 Faute de recourir à des schèmes de représentation symbolique d'ordre iconique ou imagos, la structuration personnelle des torballeurs s'effectue par régulation interactive et immanente au groupe. L'autonomie individuelle s'acquiert grâce à l'autonomie du groupe. La maîtrise des affects et des conventions sociales se fait ainsi par auto-régulation collective.

87 Cette tentative de déstigmatisation interne vient d'une volonté de construction d'une identité de groupe spécifique. La vie collective que ces joueurs partagent au sein de ce groupe, ancre chaque individu dans un univers affectif d'appartenance, un peu secret, un peu sacré. Ce phénomène évoque les rites initiatiques qui se situent toujours dans des lieux de ressourcement écartés du reste de la communauté. Comme pour les adolescents initiés, ce fait d'appartenance identitaire autorise leur structuration, leur renaissance à la vie sociale en tant que membres à part entière de la société globale.

Conclusion générale

Cette recherche montre que, dans un premier temps, la volonté individuelle d'assimilation est freinée par la déficience visuelle ou la cécité. Les sujets atteignent, dans un second temps, son dépassement par le recours à l'insertion communautaire dans un groupe sportif spécifique et structuré. Ils revendiquent alors la reconnaissance sociale de leur pratique pour vivre en coexistence avec les voyants. Cette étape est susceptible, dans un troisième temps, d'aboutir à un désir réel d'intégration respectant les spécificités de l'individu dans sa volonté de communication et de participation à la vie sociale. Ainsi l'intégration de certaines catégories de personnes dites "handicapées", dans une structure sportive, parait passer par la pratique d'une 
activité sportive spécifique. Facteur de reconnaissance de leur communauté, elle permet d'exploiter et d'assimiler leurs qualités de véritables sportifs.

On ne peut alors qu'inciter à la création de nouvelles disciplines sportives propres à chaque type de handicap, et non pas à des adaptations excessives d'A.R.S. de personnes valides. Considérant que la comparaison les performances des sportifs valides et handicapés n'a guère de sens, cette transformation des représentations appelle plutôt une spécification des pratiques sportives que leur facilitation.

La valorisation des individus portant un stigmate ne devrait donc pas se faire par l'amoindrissement ou la réduction des difficultés inhérentes aux sports de personnes valides, mais par l'invention d'A.P.S. qui leur seraient spécifiques. Une apparente ségrégation, au départ, pourrait bien alors se révéler être le gage, à terme, d'un lien social renforcé.

\section{BIBLIOGRAPHIE}

Anzieu D. (1975) Le groupe et l'inconscient. Paris, Dunod.

Alderman R. B. (1983) Manuel de psychologie du sport. Paris, Editions Vigot.

Broyer G., (1995) Pratique physique et sportive et réhabilitation psychologique et sociale. Sport et (re)construction identitaire des personnes handicapées, Bilard J. et Durand M. (dir) Psychologie du sport, Paris, Editions Revue EPS.

Bullinger A. (1992) Un corps à habiter, ou le développement sensorimoteur du bébé aveugle et malvoyant, Activités Physiques adaptées aux handicapés de la vue, 2e Congrès Européen, GRICCA, Bruxelles, Mai 1992.

Garel J-R et Siros M. (1992) Enseigner les sports collectifs aux aveugles. Ministère de l'E.N., CNEFEI, Suresnes.

Goffman E. (1975) Stigmate, les usages sociaux des handicaps. Paris, Editions de Minuit.

Hatwell Y. (1966) Privation sensorielle et intelligence. Paris, PUF.

Labridy F. (1993) Acte sportif, acte psychanalytique, Quel Corps? Sciences humaines cliniques et pratiques corporelles, $43-44$.

Le Breton D. (1995) Pratique physique et sportive et réhabilitation psychologique et sociale. Aspects anthropologiques des Activités Physiques Adaptées, Bilard J. et Durand M. (dir) Psychologie du sport, Paris, Editions Revue EPS, 159.

Levêque M. et Heuzé J.-P. (1995) Pratique physique et sportive et réhabilitation psychologique et sociale. L'environnement relationnel du sportif, Bilard J. et Durand M. (dir) Psychologie du sport, Paris, Editions Revue EPS.

Marcellini A. (1991) Sport, stigmate et intégration sociale des personnes handicapées: contribution à l'étude des stratégies de déstigmatisation. Thèse STAPS, Université de Montpellier 1. 
Marcellini A. (1995) Approche multiclimentionnelle de l'équipe sportive de haut-niveau : de la cohésion à la régulation, Bilard J. et Durand M. (dir) Psychologie du sport, Paris, Editions Revue EPS.

Moscovici S. (Il 991) Psychologie des minorités actives. Paris, PUE

Schepens C. (1992) Vous avez dit verbalisme? Activités Physiques adaptées aux handicapés de la vue, 2e Congrès Européen, GRICCA, Bruxelles, Mai 1992.

Stiker H.-J, (1982) Corps infirmes et sociétés. Paris, Aubier.

Tap P (1988) La société pygmalion? Intégration sociale et réalisation de la personne -. Paris, Editions Dunod.

Villey P (Il 922) La pédagogie des aveugles. Paris, Félix Alcan.

\section{RÉSUMÉS}

Cette étude est consacrée au torball - sport collectif officiel et spécifique - et aux non-voyants et mal-voyants. L'objectif de cette recherche est de montrer l'évolution diachronique des sujets et le paradoxe de cette activité sportive. De par leur cécité ou leur déficience visuelle, ces personnes sont exclues de la culture sportive des voyants. Alors, elles mettent en œuvre un processus ségrégatif très identitaire, d'abord individuel puis collectif, afin d'obtenir dans un second temps la reconnaissance sociale de leur pratique pour développer ensuite un processus de légitimation. Le torball présente ainsi les attributs des sports collectifs. Aveugles et déficients visuels sportifs sont à leur tour investis des attributs des sportifs ordinaires et de ceux des sportifs de haut niveau voyants. Enfin, ils acquièrent par leur volonté de légitimation la possibilité de s'intégrer et de changer les représentations sociales de la population.

This study is based on torball -a specific and official collective sport- and on visually deficient people. The aim of this research is to establish the paradoxical evolution of the players. Because of their handicap, they are excluded from the majority of the sporting practices. Therefore, they begin a process of individual, then collective identification in order to obtain social recognition and then legitimisation. So, the torball is compared with the other collective sports. The visually deficient people are also compared with the other sportsmen and champions. Finally, thanks to their wish of legitimisation, they acquire the ability to integrate themselves and to change the social representations of the other people.

\section{INDEX}

Keywords : torball, ségrégation, intégration, visually deficient people, segregation, collective identity, integration, process of transformation

Mots-clés : déficients visuels, identité de groupe, processus de transformation

\section{AUTEURS}

ODILE DELEUZE

Professeur EPS à l'Institut Arc-En-Ciel des Jeunes Aveugles et Amblyopes de Marseille 


\section{GILLES BUI-XUÂN}

Faculté des Sciences du Sport et de l'Education Physique. Université Montpellier 1.

Equipe « Corps et Culture» 